\title{
Wakefields in photonic crystal cavities
}

\author{
Gregory R. Werner, ${ }^{1, *}$ Carl A. Bauer, ${ }^{1}$ and John R. Cary ${ }^{1,2}$ \\ ${ }^{1}$ Center for Integrated Plasma Studies, University of Colorado at Boulder, Boulder, Colorado 80309, USA \\ ${ }^{2}$ Tech-X Corporation, Boulder, Colorado 80303, USA
}

(Received 3 February 2009; published 24 July 2009)

\begin{abstract}
Simulations of charged particle beams in accelerator cavities show that a two-dimensional photonic crystal can be used to create a three-dimensional hybrid cavity with a single operating mode and no significant higher order modes, greatly reducing long-range wakefields. Optimizing the photonic crystal cavity to reduce radiation leakage in the desired mode (of a finite-sized structure) yields even lower wakefields. Wakefields in photonic crystal cavities are compared to those in metal pillbox cavities.
\end{abstract}

\section{INTRODUCTION}

The band gap of a photonic crystal (PhC) can be exploited to confine electromagnetic fields, as in an rf resonant accelerator cavity [1-4]. PhC cavities can confine modes that have frequencies within the band gap without trapping waves at frequencies outside the band gap; consequently, a PhC cavity can be designed to have only a single mode, without any higher order modes (HOMs). In contrast, closed metal cavities have an infinite number of HOMs.

In cavities used to accelerate charged particle beams, the interaction between the beam and one mode-the accelerating mode-is desirable, while interaction with HOMs generally degrades the beam quality. Although larger beam tubes and specialized damping schemes can mitigate the effects of HOMs in metal cavities, PhC cavities intrinsically avoid HOM problems.

Since three-dimensional (3D) $\mathrm{PhC}$ cavities can be difficult to construct, hybrid cavities using 2D PhCs could offer a practical alternative, while still inhibiting the excitation of undesirable modes. We measure the extent to which various modes can be excited by computing the wakefields of a small bunch of relativistic electrons in different cavities without beam tubes: a metal pillbox (cylindrical) cavity, a hybrid cavity based on a 2D PhC, and a hybrid cavity based on a similar optimized structure. Dipole wakefields for hybrid 2D PhC using metal elements for the $\mathrm{PhC}$ cavities have been computed by others $[5,6]$, under somewhat different conditions. Before showing the resulting wakefields, we briefly review photonic crystal cavities and the computation of wakefields.

In this paper we aim to calculate general properties regarding wakefields of hybrid cavities (that trap fields in two dimensions using a 2D PhC, and in the third dimension using metal boundaries); therefore we have stripped the cavities of details such as beam tubes and rf input couplers.

\footnotetext{
*Greg.Werner@colorado.edu
}

The results of this paper can in principle be scaled to any frequency; however, based on ease of construction, we currently imagine such structures restricted to $\mathrm{MHz}-\mathrm{THz}$ frequencies (the low $\mathrm{GHz}$ range allows the easiest construction). Practical designs for $\mathrm{PhC}$ cavities similar to those considered here, but with input couplers, beam tubes, and/or multiple cells (so the structure can be operated in a traveling-wave mode) can be found in $[3,4,7]$, which describe actual experiments. A review of a different type of $\mathrm{PhC}$ accelerating cavity-intended to be driven by pulsed lasers at optical frequencies — can be found in Ref. [8].

\section{PHOTONIC CRYSTAL CAVITIES}

A photonic crystal $(\mathrm{PhC})$ is a regular lattice of identical light-scattering objects (e.g., a lattice of metal or glass spheres); some photonic crystals have a band gap-a frequency range devoid of any propagating electromagnetic states [9]. In other words, waves with frequencies in the band gap cannot propagate through the PhC. Band gaps and other interesting $\mathrm{PhC}$ phenomena occur at wavelengths on the order of the lattice spacing.

A photonic crystal with a band gap is a frequencyselective mirror, reflecting radiation with frequencies within a band gap, while allowing frequencies outside the band gap to travel through the crystal. Radiation impinging upon a (semi-infinite) photonic crystal will be completely reflected if the radiation has a frequency within the band gap, because it cannot propagate through the crystal; the fields penetrating the crystal will be evanescent, transporting no energy. Frequencies outside the band gap can travel through the crystal, so they will not be completely reflected at the interface.

A cavity (or more generally, a lattice defect) within a $\mathrm{PhC}$ can trap fields that have frequencies within the band gap, since those fields cannot escape through the surrounding crystal. The cavity can therefore support resonant modes with frequencies in the band gap. The cavity cannot have resonances (i.e., HOMs) outside the band gap because any such fields would simply propagate away. If a $\mathrm{PhC}$ 


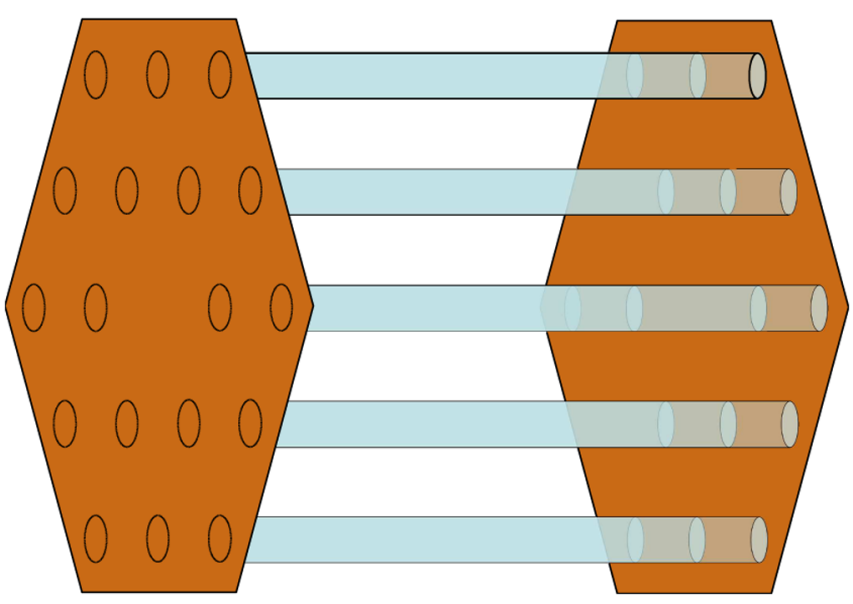

FIG. 1. (Color) A PhC cavity with dielectric rods in a triangular lattice, with the center rod removed, and metal ends. The beam travels down the center of the cavity, where the removed rod was.

cavity has just one mode within the band gap, then it has no HOMs at all.

Because three-dimensional $\mathrm{PhCs}$ can be difficult to build, we examined a hybrid cavity, using a twodimensional $\mathrm{PhC}-\mathrm{a}$ triangular lattice of alumina rods $\left(\epsilon_{r}=10\right)$ - terminated by metal end plates to confine the fields in the third dimension (Fig. 1). The cavity was formed by removing one dielectric rod. A similar cavity has been built and tested experimentally [4].

The band gap of the 2D PhC applies to wave propagation in the 2D plane. As long as we consider only 2D solutions, the 2D PhC cavity has no HOMs. The trapped mode in the 2D PhC cavity (see Fig. 2) also exists in the 3D hybrid cavity because the fields are consistent with the boundary conditions imposed by the metal end plates (the electric

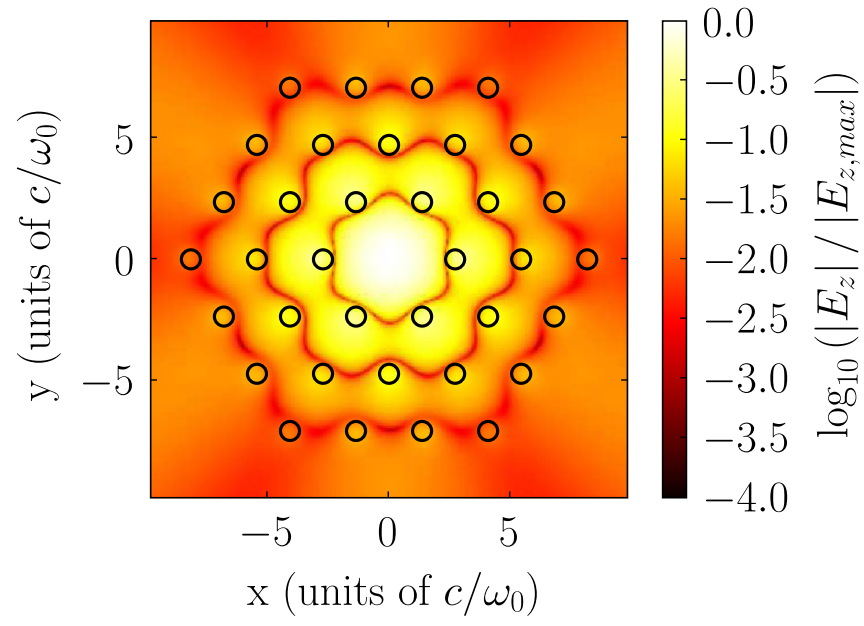

FIG. 2. (Color) Axial electric field strength for a $\mathrm{PhC}$ cavity in a triangular lattice of dielectric rods. $\omega_{0}$ is the resonant frequency and $c$ is the speed of light. The 36 black rings indicate the positions and diameters of the dielectric rods.

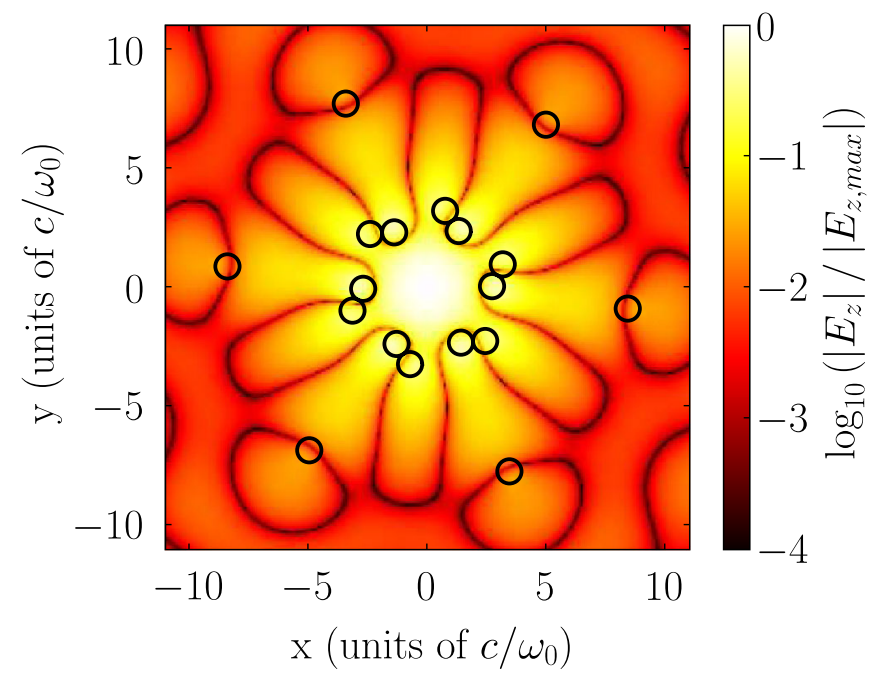

FIG. 3. (Color) Axial electric field strength in the optimized PhC cavity. The 18 black rings indicate the positions and diameters of the dielectric rods (cf. Fig. 2).

field is perpendicular and the magnetic field is parallel to the plates). However, the 3D structure could have HOMs involving out-of-plane propagation. For example, there are trapped modes that are guided in the dielectric rods by total internal reflection.

The field pattern of the accelerating mode of the hybrid cavity is the same as the trapped mode of the $2 \mathrm{D} \mathrm{PhC}$. The electric field strength of this mode, in a PhC cavity truncated to three hexagonal rings of rods (36 rods), is depicted in Fig. 2. Because the crystal is truncated after three rings of rods, the resonant mode is imperfectly confined; in this case, the $Q$ factor due to radiation leaking out through the crystal (assuming perfect absorbers outside the crystal, and ignoring dielectric losses) is $Q_{\mathrm{rad}}=650$. See Ref. [10] for details about measuring the $Q$ factor.

Previous work [10] has shown that a truncated photonic crystal is not optimal for trapping fields in a cavity. By optimizing the location of individual rods, another photonic structure with only 18 rods $\left(\epsilon_{r}=10\right)$ has been found to limit radiation losses so that $Q_{\text {rad }}=11000$. Although this "optimized PhC cavity" traps the fundamental mode better, it turns out (accidentally) to be even less susceptible to HOMs. The electric field strength of the resonant mode in the optimized $\mathrm{PhC}$ cavity is shown in Fig. 3.

Having examined the accelerating mode by considering the 2D PhC cavity, we next explore what other modes in the 3D hybrid cavity might be excited by a charged beam.

\section{WAKE POTENTIAL COMPUTATION}

We used the wake potential to quantify the interaction between cavity fields and a relativistic electron beam in the cavity (cf. [11]), and we calculated the wake potential through computer simulation of a beam exciting a cavity (see also [12]). 
When a point charge $q_{b}$ ( $b$ for "beam") travels through a cavity, it creates an electric field $\mathbf{E}(r, \theta, z, t)$ (and also a magnetic field); the wake potential describes the momentum change of a test charge due to those fields. If a charge $q_{b}$ travels at light speed along the path $(r, \theta, z)(t)=$ $\left(r_{b}, \theta_{b}, c t\right)$, creating an electric field with $z$ component $E_{z}(r, \theta, t, z)$, its longitudinal wake potential is

$W_{z}\left(r_{b}, \theta_{b}, r_{t}, \theta_{t}, s\right) \equiv-\frac{1}{q_{b}} \int d z E_{z}\left[r_{t}, \theta_{t}, z, t=(z+s) / c\right]$.

If a test charge $q_{t}$ travels along the path $(r, \theta, z)(t)=$ $\left(r_{t}, \theta_{t}, c t+s\right)$, its longitudinal momentum change due to the fields created by $q_{b}$ is

$$
\Delta p_{z}\left(r_{t}, \theta_{t}, s\right)=-q_{t} q_{b} W_{z}\left(r_{b}, \theta_{b}, r_{t}, \theta_{t}, s\right)
$$

(the test charge is assumed to be highly relativistic, so its speed remains constant even as its momentum changes). By convention, positive $W_{z}$ corresponds to a loss in momentum.

We consider cavities without beam tubes, so the path of integration will extend from $z=0$ to $z=\ell$, the length of the cavity. Beam tubes have a considerable effect on the wakefields [13] (Sec. 3.2.5, "Explicit Expressions of Impedances and Wake Functions") [14] (Sec. 15.6), with larger beam tubes generally diminishing wakefields; because the size of the beam tube is affected by factors other than the desire for lower wakefields, we felt it best to compare cavities without beam tubes.

In cylindrically symmetric structures (such as pillbox or elliptical accelerator cavities), it is helpful to decompose wakefields into azimuthal harmonics, i.e., fields with dependence $\cos (m \theta)$ and $\sin (m \theta)$ for $m=0,1, \ldots$ :

$$
\begin{aligned}
W_{z}\left(r_{b}, r_{t}, \theta_{t}, s\right)= & \sum_{m=0}^{\infty} W_{z, m}\left(r_{b}, r_{t}, s\right) \cos \left(m \theta_{t}\right) \\
& +W_{z, s m}\left(r_{b}, r_{t}, s\right) \sin \left(m \theta_{t}\right),
\end{aligned}
$$

where we have chosen the beam to be at $\theta_{b}=0$; if the structure has symmetry with respect to $\theta \rightarrow-\theta$, then $W_{z, s m}=0$. Of course, the wake potential can always be decomposed as above, but in structures with cylindrical symmetry and infinitely long beam tubes, the azimuthal components of the wakefield have a particularly simple dependence on $r_{b}$ and $r_{t}$ (when they are smaller than the beam tube radius) [15]:

$$
W_{z, m}\left(r_{b}, r_{t}, s\right) \propto r_{b}^{m} r_{t}^{m} \quad W_{z, s m}=0 .
$$

Reference [16] shows that this simple form depends on the fields at $z= \pm \infty$ being the fields of a charge traveling in an infinitely long beam tube; in a closed cavity, the fields at the ends of integration (at $z=0$ and $z=\ell$ ) between $r_{b}$ and $r_{t}$ upset the simple dependence on $r_{b}$ and $r_{t}$, but $W_{z, m}$ still approximately assumes the above form for $r_{b}$ and $r_{t}$ close to the axis of symmetry.
To calculate $W_{z, m}\left(r_{b}, r_{t}, s\right)$, we used the electromagnetic particle-in-cell (PIC) module of VORPAL [17]. We excited a cavity using a current bunch at $r_{b}$ (and $\theta_{b}=0$ ), with a Gaussian width $\sigma_{z}$ in the longitudinal direction; the current bunch traveled at highly relativistic speed and was (artificially) unaffected by the fields it generated. At each time step, we injected highly relativistic test particles (with charges too small to affect the cavity fields) with radius $r_{t}$ at regularly spaced angles $\theta_{t}$, and recorded the momentum change of each test particle. We thus measured $\Delta p_{z}\left(r_{t}, \theta_{t}, s\right)$, which, after decomposition into azimuthal harmonics, yielded $W_{z, m}\left(r_{b}, r_{t}, s\right)$.

Each cavity was designed to have its $\mathrm{TM}_{010}$-like mode at $10 \mathrm{GHz}$; the length of each cavity was one-half wavelength at that frequency, $\ell=15.0 \mathrm{~mm}$. We chose a bunch length $\sigma=2 \ell / 25$. Following the advice of Ref. [13] (Sec. 3.2.3, "Impedance Calculation, Time Domain"), we chose the cell length $\Delta z<\sqrt{\sigma^{3} / \ell}$, resulting in $\Delta z=0.333 \mathrm{~mm}$ and $\Delta x=\Delta y=0.336 \mathrm{~mm}$.

\section{RESULTING WAKEFIELDS}

Figure 4 shows the monopole wake potential $W_{z, 0}(s)$ for $r_{b}=r_{t}=0.67 \mathrm{~mm}$ in the three different cavities: metal pillbox, PhC (depicted in Fig. 2), and optimized $\mathrm{PhC}$ (depicted in Fig. 3). The drive current that excited the wake is displayed at the bottom of the graphs. Compared to the pillbox cavity, the $\mathrm{PhC}$ and optimized $\mathrm{PhC}$ cavities clearly have wakefields more dominated by the fundamental $10 \mathrm{GHz}$ mode. Through comparison with the wakefields at $r_{t}=2(0.67) \mathrm{mm}$, we verified that $r_{t}$ is small enough that $W_{z, m} \propto r_{t}^{m}$ (at least for $m=0,1$, and 2). This means that the $W_{z, m}$ we have calculated can be used to find the transverse wakefields, using the Panovsky-Wenzel theorem [11], so the strength of the transverse wakefields is proportional to the strength of the longitudinal wakefields.

The initial spike in the wakefield is the same for all cavities. Since all cavities are identical up to a radius of about $10 \mathrm{~mm}$, a test particle cannot "see" the difference between cavities until a wave has had time to travel from the drive beam out to radius $10 \mathrm{~mm}$ and reflect back to the test charge. The initial spike would be affected by the choice of beam tube radius, which is zero in this case.

Because the $\mathrm{PhC}$ and optimized $\mathrm{PhC}$ cavities are not cylindrically symmetric, the wake potential depends on the $\theta_{b}$, the angular position of the beam, and not just on $\theta_{t}-$ $\theta_{b}$. However, contribution of the various modes to the wake potentials does not significantly change as $\theta_{b}$ changes.

The optimized PhC cavity does not have $\theta \rightarrow-\theta$ parity symmetry, and the PhC cavity has parity symmetry only when the beam is at the same angle as a rod, or halfway between two rods. In the absence of this parity symmetry, the wakefields can have sine terms, $W_{z, s m} \neq 0$ (of course, there is never any sine term for $m=0$ ). For small $s$, the sine terms are negligible; for larger $s$, the sine terms resemble the cosine terms in strength and character. 


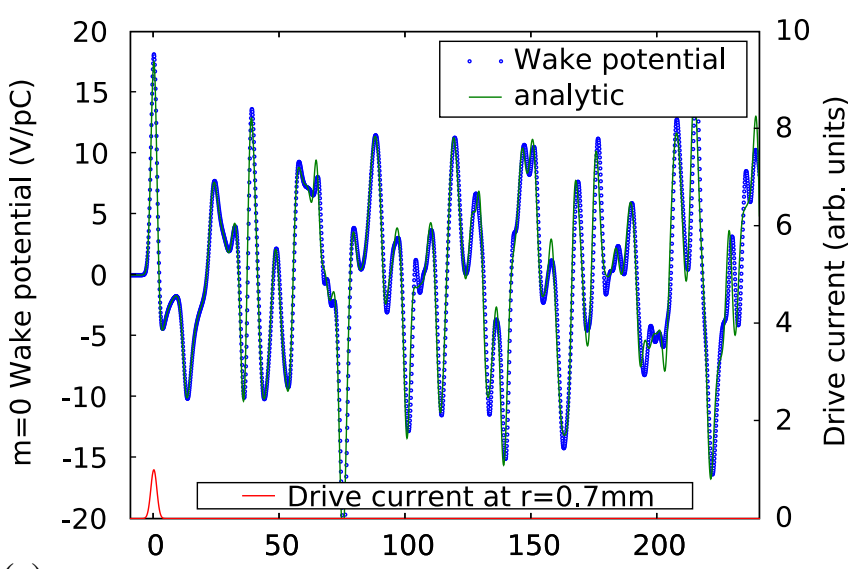

(a) $\mathrm{s}(\mathrm{mm})$
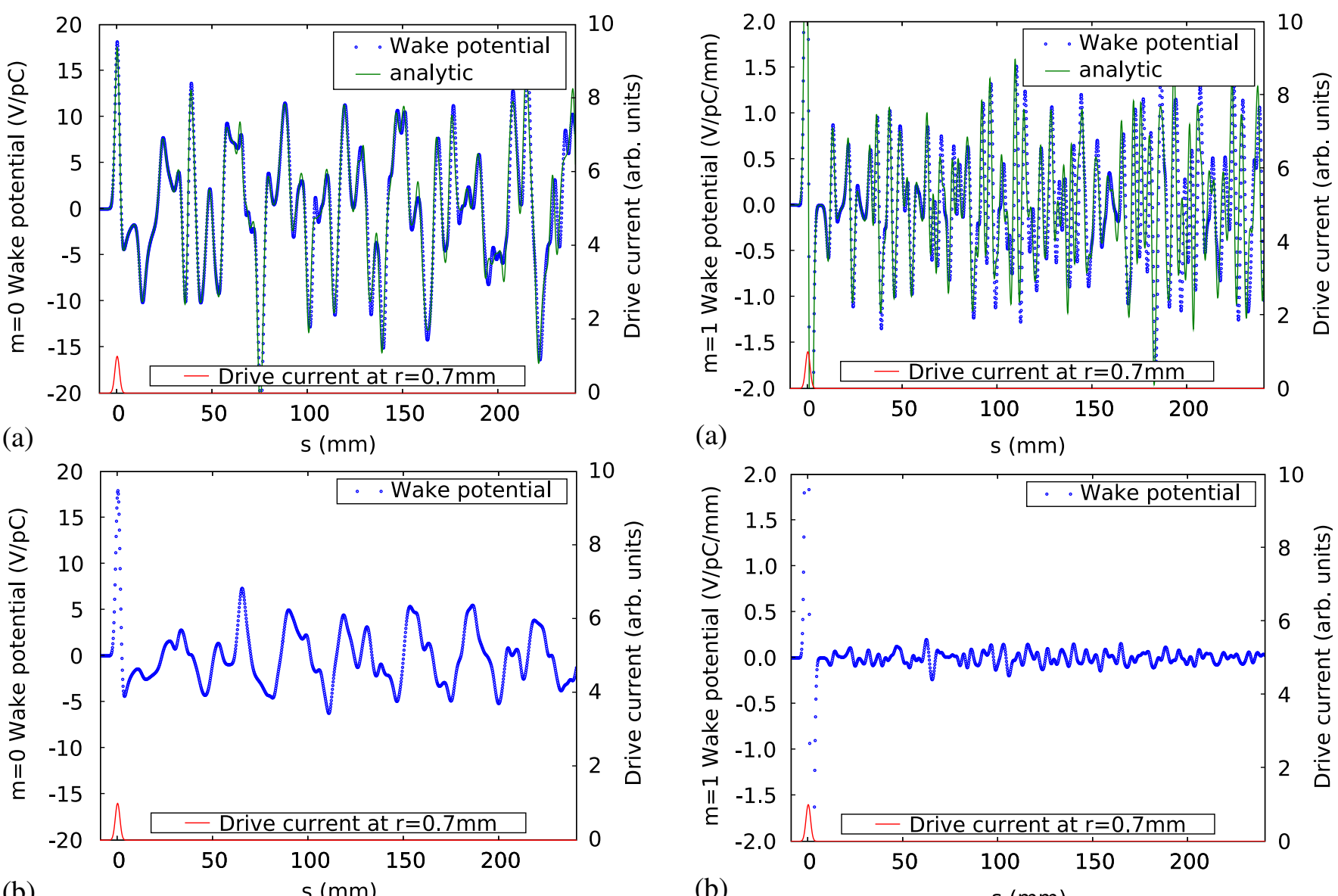

(a)

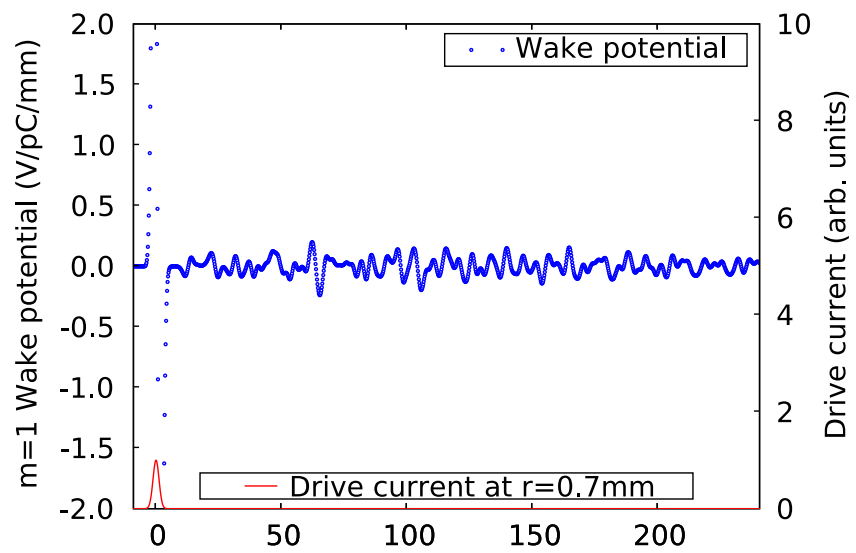

(b)
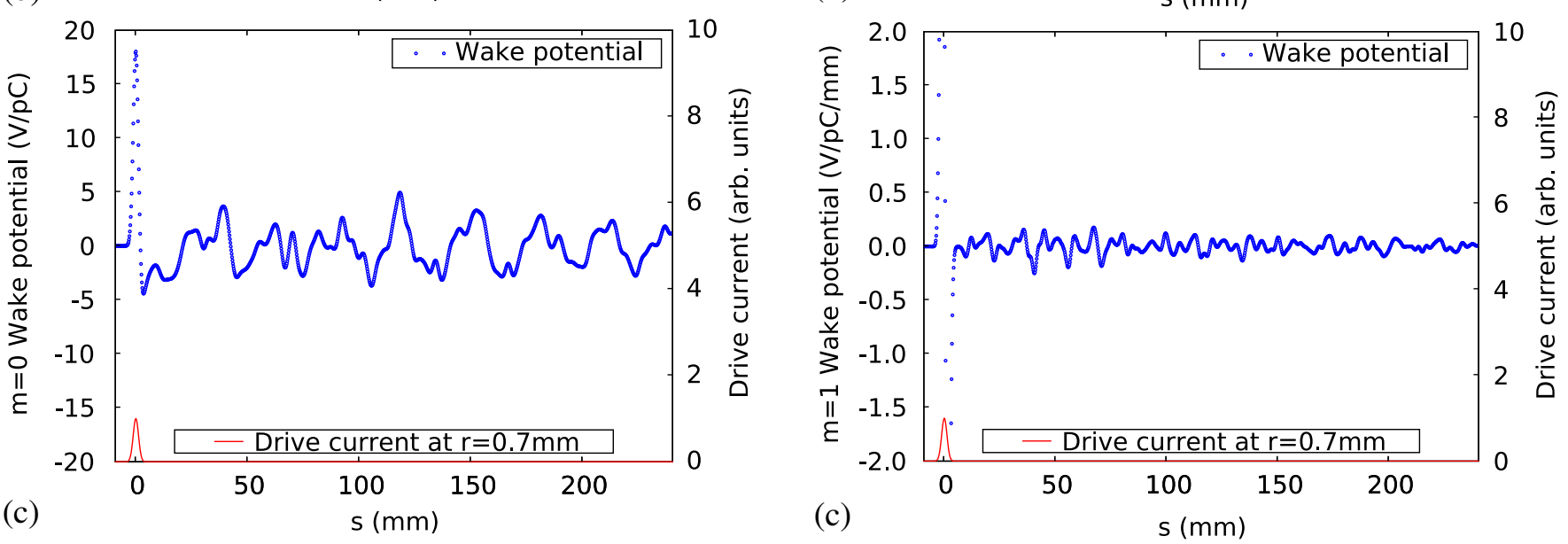

FIG. 4. (Color) Monopole wake potentials in the (a) closed pillbox cavity, (b) $\mathrm{PhC}$ cavity, and (c) optimized $\mathrm{PhC}$ cavity.

Figure 5 shows the dipole wake potential divided by the test particle radius, $W_{z, 1}(s) / r_{t}$, for $r_{b}=r_{t}=0.67 \mathrm{~mm}$. That close to the axis, $W_{z, 1}$ is approximately proportional to $r_{b} r_{t}$. Compared to the pillbox cavity, the $\mathrm{PhC}$ and optimized $\mathrm{PhC}$ cavities have much-reduced dipole modes; multipole modes are similarly reduced.

For the pillbox cavity, analytic wake potentials [12] are overlaid over simulated wake potentials to show the nu-

FIG. 5. (Color) Dipole wake potentials (divided by $r_{t}$ ) in the (a) closed pillbox cavity, (b) PhC cavity, and (c) optimized $\mathrm{PhC}$ cavity.

merical accuracy with the chosen resolution. The simulated wake potential has excellent qualitative agreement with the analytic result, and the quantitative agreement is very good, with the exceptions that the simulated potential sometimes blurs small-scale features such as sharp peaks, and there is a growing phase lag between the two results due to errors in the cavity frequency caused by discretiza- 
tion (increasing the simulation resolution reduces these discrepancies). Although the numerically computed wakefields are essentially produced by a beam that is one cell in cross section (rather than by a pencil-thin beam), comparisons between analytic and computed wakefields in the

(a)

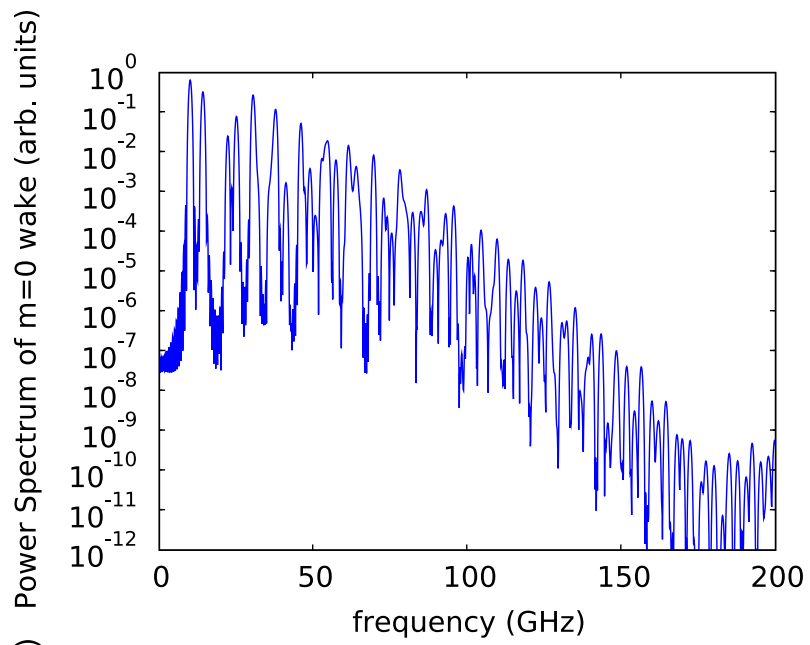

(b)

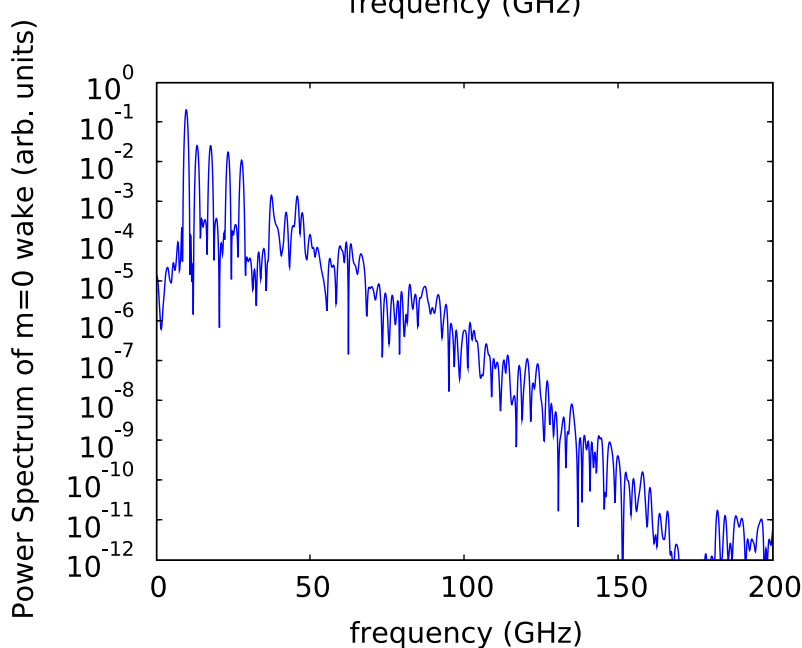

(c)

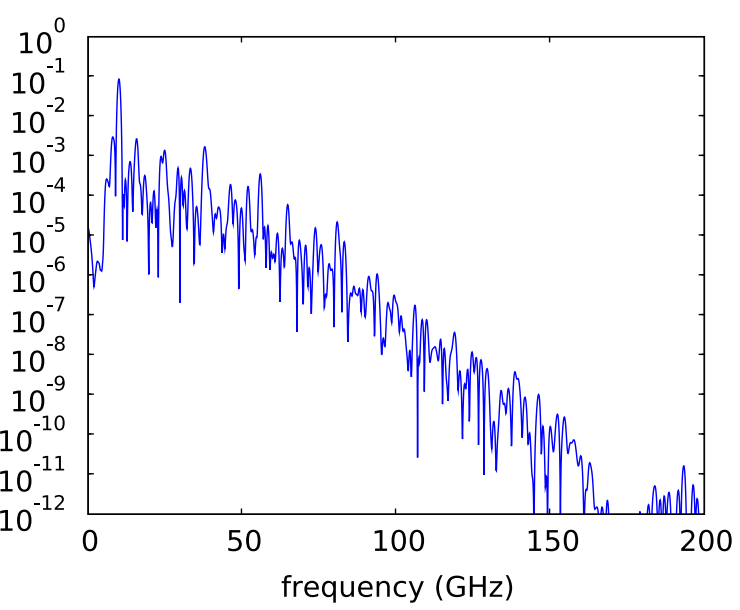

FIG. 6. (Color) Power spectra of the monopole wakes in the (a) closed pillbox cavity, (b) PhC cavity, and (c) optimized $\mathrm{PhC}$ cavity. pillbox cavity show that the beam cross section has a negligible effect; the effects of frequency errors and poor simulation of high frequencies due to the coarse resolution play a larger role [they account for all visible differences in Fig. 4(a)].

To get an idea of how monopole HOMs are suppressed in $\mathrm{PhC}$ and optimized $\mathrm{PhC}$ cavities, we have graphed the power spectrum of the monopole wake potentials $W_{z, 0}(s)$ (Fig. 6), to separate the desired from the undesired modes. Whereas the pillbox cavity has HOMs excited at nearly the same level as the fundamental mode, the $\mathrm{PhC}$ cavity shows a significant reduction in $\mathrm{HOMs}$, and the optimized $\mathrm{PhC}$ suppresses HOMs even more.

In the closed metal pillbox cavity, there is no dissipation (in our simulation, which neglects wall losses), so modes excited by the beam ring forever. The $\mathrm{PhC}$ and optimized $\mathrm{PhC}$ cavities absorb all waves that hit the simulation boundary, but also neglect losses in the dielectric rods and metal walls. Because fields decay in the PhC cavities but not in the pillbox cavity, the power spectra depend on the period of time over which the time series are recorded before taking the Fourier transform; if we recorded field values for a longer time, the peak heights would not change much for the pillbox cavity, while the peak heights for the other cavities would decrease as the time increases (although the peak for the fundamental mode would decrease relatively slowly).

We also simulated wakefields in $\mathrm{PhC}$ cavities with two rings of rods (18 rods) and four rings (60 rods); although the $Q$ factor of the accelerating mode increases greatly when rods are added, the wakefields looked essentially the same (at least out to $s=250 \mathrm{~mm}$ ).

\section{CONCLUSION}

Undesirable interactions between a beam and HOMs can be greatly reduced by using an rf accelerator cavity designed from a 2D photonic crystal. When a charge bunch passes through a photonic crystal cavity, it excites mainly the desired accelerating mode. HOMs are even more suppressed in a structure based on a 2D photonic crystal, but optimized to trap the accelerating mode more effectively.

$\mathrm{PhC}$ cavities may also be useful for rf sources; our results demonstrate that sending a charged bunch through a PhC cavity results in more efficient transfer of energy to a single mode, compared to a metal pillbox cavity.

\section{ACKNOWLEDGMENTS}

To compute wakefields we used the simulation framework VORPAL, which was developed with support of the Offices of FES, HEP, and NP of the Department of Energy, the SciDAC program, AFOSR, JTO, Office of the Secretary of Defense, and the SBIR programs of the Department of Energy and Department of Defense. We would also like to acknowledge assistance from the rest 
of the VORPAL team: T. Austin, G. I. Bell, D. L. Bruhwiler, R. S. Busby, J. Carlsson, B. M. Cowan, D. A. Dimitrov, A. Hakim, J. Loverich, P. Messmer, P. J. Mullowney, C. Nieter, K. Paul, S. W. Sides, N.D. Sizemore, D. N. Smithe, P. H. Stoltz, S. A. Veitzer, D. J. Wade-Stein, M. Wrobel, N. Xiang, and W. Ye.

[1] N. Kroll, D. R. Smith, and S. Schultz, in 1992 Advanced Accelerator Concepts, AIP Conf. Proc. No. 279 (AIP, New York, 1993), p. 197.

[2] D. R. Smith, D. Li, D. C. Vier, and S. Schultz, in 1996 Advanced Accelerator Concepts, AIP Conf. Proc. No. 398 (AIP, New York, 1997), p. 518.

[3] E. I. Smirnova, A. S. Kesar, I. Mastovsky, M. A. Shapiro, and R. J. Temkin, Phys. Rev. Lett. 95, 074801 (2005).

[4] M. R. Masullo, A. Andreone, E. Di Gennaro, S. Albanese, F. Francomacaro, M. Panniello, V. G. Vaccaro, and G. Lamura, Microw. Opt. Technol. Lett. 48, 2486 (2006).

[5] D. Li, N. Kroll, D. R. Smith, and S. Schultz, in 1996 Advanced Accelerator Concepts, AIP Conf. Proc. No. 398 (AIP, New York, 1997), p. 528.

[6] N. Kroll, S. Schultz, D. R. Smith, and D. C. Vier, in Proceedings of the Particle Accelerator Conference, New York, 1999 (IEEE, New York, 1999), p. 830.
[7] E. Di Gennaro, S. Savo, A. Andreone, G. Castaldi, V. Pierro, and M. R. Masullo, Appl. Phys. Lett. 93, 164102 (2008).

[8] B. M. Cowan, R. L. Byer, E. Colby, R. J. England, C. McGuiness, R. J. Noble, T. Plettner, C. M. S. Sears, R. H. Siemann, and J. E. Spencer, in 2008 Advanced Accelerator Concepts, AIP Conf. Proc. No. 1086 (AIP, New York, 2008), p. 56.

[9] J. D. Joannopoulos, Photonic Crystals: Molding the Flow of Light (Princeton University Press, Princeton, NJ, 1995).

[10] C. A. Bauer, G. R. Werner, and J. R. Cary, J. Appl. Phys. 104, 053107 (2008).

[11] P. B. Wilson, SLAC Technical Report No. SLAC-PUB4547, 1989.

[12] G. R. Werner, arXiv:0906.1007.

[13] Handbook of Accelerator Physics and Engineering, edited by A.W. Chao and M. Tigner (World Scientific, Singapore, 1999).

[14] H. Padamsee, J. Knobloch, and T. Hays, $R F$ Superconductivity for Accelerators (Wiley, New York, 1998).

[15] T. Weiland, Nucl. Instrum. Methods 216, 31 (1983).

[16] O. Napoly, Y.H. Chin, and B. Zotter, Nucl. Instrum. Methods Phys. Res., Sect. A 334, 255 (1993).

[17] C. Nieter and J.R. Cary, J. Comput. Phys. 196, 448 (2004). 